\title{
Safety and clinical efficacy of BCMA CAR-T-cell therapy in multiple myeloma
}

\author{
Gils Roex ${ }^{1 \dagger}$, Marijke Timmers ${ }^{2 \dagger}$, Kristien Wouters $^{3}$, Diana Campillo-Davo ${ }^{1}$, Donovan Flumens ${ }^{1}$, \\ Wilfried Schroyens ${ }^{2}$, Yiwei Chu ${ }^{4}$, Zwi N. Berneman², Eva Lion' ${ }^{1}$, Feifei Luo ${ }^{4,5}$ and Sébastien Anguille ${ }^{1,2^{*}}$ (D)
}

\begin{abstract}
Background: B-cell maturation antigen (BCMA)-targeted chimeric antigen receptor (CAR)-T-cell therapy is an emerging treatment option for multiple myeloma. The aim of this systematic review and meta-analysis was to determine its safety and clinical activity and to identify factors influencing these outcomes.

Methods: We performed a database search using the terms "BCMA," "CAR," and "multiple myeloma" for clinical studies published between 01/01/2015 and 01/01/2020. The methodology is further detailed in PROSPERO (CRD42020125332).
\end{abstract}

Results: Twenty-three different CAR-T-cell products have been used so far in 640 patients. Cytokine release syndrome was observed in 80.3\% (69.0-88.2); 10.5\% (6.8-16.0) had neurotoxicity. A higher neurotoxicity rate was reported in studies that included more heavily pretreated patients: $19.1 \%\left(13.3-26.7 ; P^{2}=45 \%\right)$ versus $2.8 \%\left(1.3-6.1 ; P^{2}=0 \%\right)$ $(p<0.0001)$. The pooled overall response rate was $80.5 \%$ (73.5-85.9); complete responses (CR) were observed in $44.8 \%$ (35.3-54.6). A pooled CR rate of $71.9 \%\left(62.8-79.6 ; I^{2}=0 \%\right)$ was noted in studies using alpaca/llama-based constructs, whereas it was only $18.0 \%\left(6.5-41.1 ; P^{2}=67 \%\right)$ in studies that used retroviral vectors for CAR transduction. Median progression-free survival (PFS) was 12.2 (11.4-17.4) months, which compared favorably to the expected PFS of 1.9 (1.5-3.7) months (HR 0.14; $p<0.0001)$.

Conclusions: Although considerable toxicity was observed, BCMA-targeted CAR-T-cell therapy is highly efficacious even in advanced multiple myeloma. Subgroup analysis confirmed the anticipated inter-study heterogeneity and identified potential factors contributing to safety and efficacy. The results of this meta-analysis may assist the future design of CAR-T-cell studies and lead to optimized BCMA CAR-T-cell products.

Keywords: BCMA, CAR-T, Multiple myeloma

\section{Introduction}

Multiple myeloma (MM) is defined by a malignant proliferation of plasma cells in the bone marrow (BM) $[1,2]$. As the second most common hematological malignancy after lymphomas, it accounts for $1 \%$ of all cancers [3]. Recent epidemiological studies have indicated a steady

\footnotetext{
*Correspondence: sebastien.anguille@uza.be

${ }^{\dagger}$ Gils Roex and Marijke Timmers share first authorship

${ }^{2}$ Division of Hematology and Center for Cell Therapy \& Regenerative Medicine, Antwerp University Hospital, Edegem, Belgium

Full list of author information is available at the end of the article
}

increase in the incidence and prevalence of MM, mainly attributable to the aging population and therapeutic advances improving survival [4]. Indeed, over the past two decades, the landscape of myeloma treatment has dramatically changed with the advent of several novel therapies, including monoclonal antibodies (mAbs) [5].

Recently, chimeric antigen receptor (CAR)-T-cell immunotherapy has entered the clinical trial arena $[6$, 7]. CAR-T cells are autologous lymphocytes collected by leukapheresis and genetically modified (most often by lentiviral or retroviral transduction) to express a CAR. Following ex vivo expansion, the cells are then reinfused original author(s) and the source, provide a link to the Creative Commons licence, and indicate if changes were made. The images or other third party material in this article are included in the article's Creative Commons licence, unless indicated otherwise in a credit line to the material. If material is not included in the article's Creative Commons licence and your intended use is not permitted by statutory regulation or exceeds the permitted use, you will need to obtain permission directly from the copyright holder. To view a copy of this licence, visit http://creativecommons.org/licenses/by/4.0/. The Creative Commons Public Domain Dedication waiver (http://creativeco mmons.org/publicdomain/zero/1.0/) applies to the data made available in this article, unless otherwise stated in a credit line to the data. 
to the patient who is usually first conditioned with lymphodepleting chemotherapy (Fig. 1) [8]. CARs are synthetic receptors that bear characteristics of a mAb and a T-cell receptor (TCR); they contain an antigen-recognition domain from a mAb (usually in single-chain variable fragment $[\mathrm{scFv}]$ format) and $\mathrm{CD} 3 \zeta$ [9]. The $\mathrm{mAb}$ part is responsible for HLA-independent binding of the CAR-T cell to a target expressed on the tumor cell surface, whereas the $\mathrm{CD} 3 \zeta$ chain triggers T-cell activation by mimicking TCR signaling. Most CAR constructs also contain one (2nd generation) or more ( $3^{\text {rd }}$ generation) costimulatory domains, such as 4-1BB or CD28 (Fig. 1) [8].

Although several antigens are undergoing clinical evaluation, B-cell maturation antigen (BCMA) has been the most popular myeloma target antigen so far [10-12]. BCMA is involved in cell survival and is expressed exclusively on the surface of B-cell lineage cells, including malignant plasma cells $[10,11]$. The impressive clinical results of CD19-targeted CAR-T cells in CD19 ${ }^{+}$hematological malignancies $[6,13,14]$ have created high expectations for CAR-T-cell therapy in other cancers [15]. However, it remains unclear whether these expectations are justified in the context of MM since doubts have recently been raised about the durability of therapeutic activity [16]. Moreover, CAR-T-cell therapy can produce potentially life-threatening toxicities, such as cytokine release syndrome (CRS) and neurotoxicity [17].

Current evidence on BCMA-targeted CAR-T-cell therapy in $M M$ is restricted to relatively small, non-randomized early phase clinical trials. Hence, at this stage, it is difficult to obtain a clear sight on the toxicity and efficacy that can be expected from this novel therapeutic approach in relapsed/refractory MM patients. To the best of our knowledge, there has been only one attempt so far to systematically aggregate the outcome data of BCMA CAR-T-cell clinical studies [18]. In that report, Gagelmann et al. included 15 studies comprising a total of 285 patients. Here, we were able to identify 27 studies involving 23 different BCMA CAR-T-cell products and a total of 640 patients, making it the most comprehensive systematic review and meta-analysis to date of the safety and clinical efficacy of BCMA-targeted CAR-T-cell therapy in MM. Moreover, this study is also the first to identify potential patient- and treatment-related factors influencing toxicity and efficacy, which helps us to understand the different outcomes between bb2121 (idecabtagene vicleucel) and LCAR-B38M (ciltacabtagene autoleucel), the two most advanced, late-stage BCMA CAR-T-cell products which are likely to receive regulatory approval in the years to come. Furthermore, controlled trials are lacking, making it challenging to assess the true progressionfree survival (PFS) benefit that is reported in individual clinical studies. In this meta-analysis, we incorporated a surrogate control arm, composed of patients treated with inactive doses of BCMA CAR-T cells. PFS data from this control population were used to determine the expected outcome in order to more accurately assess the therapeutic benefit of BCMA CAR-T-cell therapy in relapsed/ refractory MM patients.

\section{Methods}

\section{Search strategy and selection criteria}

This study involves a systematic review and meta-analysis of the risks and benefits of BCMA CAR-T-cell therapy in MM patients. Relevant clinical studies were identified by a systematic search of Web of Science (Clarivate Analytics) and PubMed/MEDLINE using the following search terms: "B-cell maturation antigen" or "BCMA," "chimeric antigen receptor" or "CAR," and "multiple myeloma." Additional records were retrieved by screening published conference abstracts of American Society of Clinical Oncology (ASCO), American Society of Hematology (ASH), European Group for Blood and Marrow Transplantation (EBMT), and European Hematology Association (EHA). All clinical trial designs (i.e., controlled and uncontrolled studies) were considered. Since the first clinical report of CAR-T-cell therapy in MM was published in 2015 [19], the search was restricted to studies published between January 1, 2015, and January 1, 2020. Only clinical trials registered on Clinicaltrials.gov (NCTnumber) or Chinese Clinical Trial Registry (ChiCTRnumber) and published in English, either as full scientific article or as abstract during the annual scientific meetings of ASCO, ASH, EBMT, or EHA, were taken into consideration. Patient data were solely extracted from these publications, and no requests for additional original patient data were made to the authors of these studies. Reviews and non-scientific publications were not used for data collection to avoid duplicate data, but were used to ensure accurate and appropriate data selection. Database searches and data collection were conducted independently by three authors (GR, MT, and SA). Data were omitted if no unanimous consensus over their inclusion was found. The PRISMA flow diagram in Fig. 2 depicts the search strategy that was followed to identify the relevant publications (Fig. 2).

\section{Data analysis}

Table S1 (Additional file 1) provides an overview of the 61 publications that were retrieved following the PRISMA flow diagram depicted in Fig. 2. Based on the clinical trial registration number (NCT-number or ChiCTR-number), the CAR-T-cell product name, and the study group (lead author and affiliation), we were able to identify 27 different studies comprising 640 unique patients. For several studies, overlapping publications were identified; to avoid 


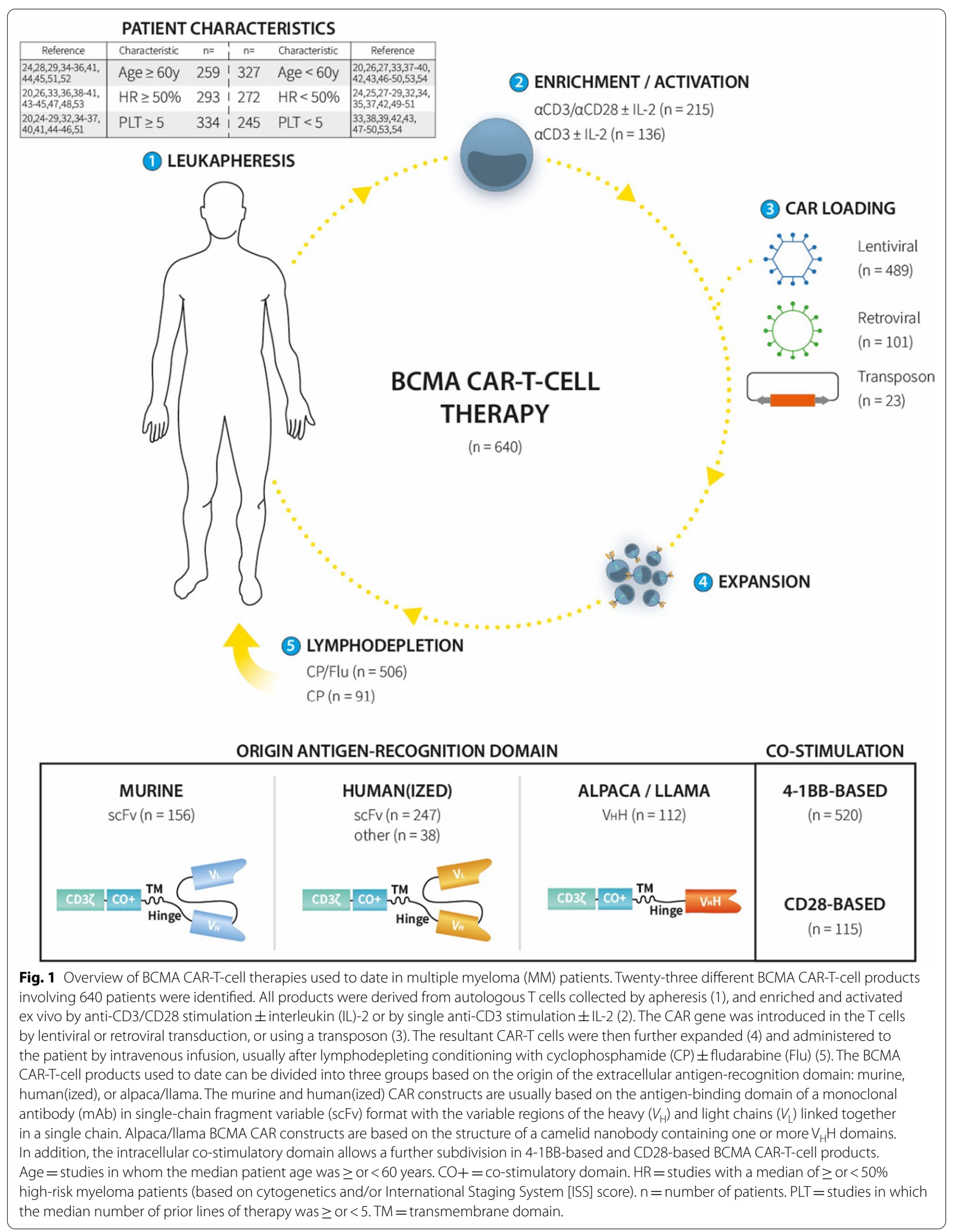




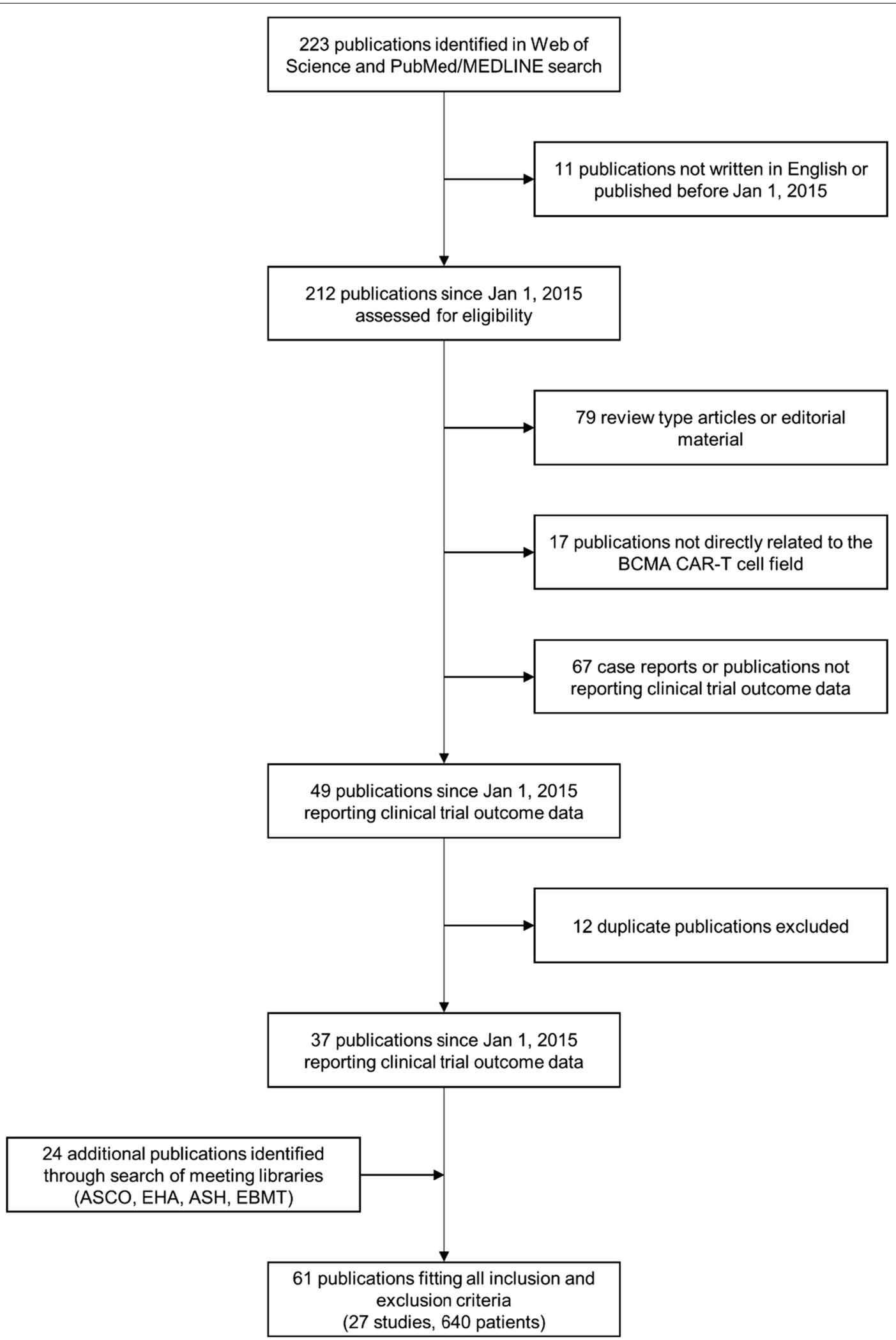

Fig. 2 Search strategy and study selection. ASCO = American Society of Clinical Oncology. ASH =American Society of Hematology. BCMA = B-cell maturation antigen. $C A R=$ chimeric antigen receptor. EBMT $=$ European Group for Blood and Marrow Transplantation. EHA $=$ European Hematology Association 
duplicate data, only the most recent and/or the largest (based on the number of included patients) records were considered (Additional file 1: Table S1). As shown in Table S1, there were two exceptions to this rule. For study NCT02546167 (CART-BCMA UPenn), we decided to use the full publication [20] rather than the meeting abstract [21]. For study NCT03661554 (BCMA nanoantibody), the latest publication involving 16 CAR-T-infused patients was not considered because outcome data were incompletely reported (only for 7 patients) [22].

Primary outcome measures were CAR-T-cell-related toxicities (i.e., CRS and neurotoxicity), and objective response rate (ORR). ORR was defined as the sum of (stringent) complete responses ([s]CR) and (very good) partial responses ([VG]PR), according to IMWG criteria [23]. Progression-free survival (PFS) was used as secondary outcome measure. We collected data on the following patient- and disease-related variables: number of patients, median age, myeloma risk (based on cytogenetics and/or International Staging System [ISS] score), and prior lines of therapy. Information on the following treatment-related variables was extracted: origin and type of the CAR antigen-recognition domain, enrichment/activation method, loading strategy, type of co-stimulatory domain, cell dosage, and lymphodepletion regimen.

We conducted a meta-analysis for proportions to estimate the overall proportion of CRS/neurotoxicity and ORR/CR. Because of the diversity between the studies, a random-effects model was used. Heterogeneity was judged by forest plots and $I^{2}$. Results are reported as proportions with $95 \%$ confidence interval (CI). Subgroup analyses were performed to assess differences between groups of studies. P values were calculated based on the between subgroups heterogeneity statistic.

Median PFS with 95\% CI was calculated from individual patient data, which were retrieved using computerized analysis of published Swimmer plots and/ or Kaplan-Meier survival curves. We verified the correctness of the retrieved data by back-checking that the calculated median PFS was identical to the published median PFS of each study. A comparative analysis was performed between CAR-T cells used at active doses with inactive doses, where an inactive dose was defined as a CAR-T cell dose that failed to produce both CRS and ORR rates of $>50 \%$. This corresponded to the patients included in the lowest dose cohorts of the following four early phase BCMA CAR-T-cell studies with a doseescalation design: NCT02658929 [24], NCT02546167 [20], NCT02215967 [25], and NCT03070327 [26]. In the absence of randomized controlled trials, the latter served as a surrogate control group to determine the expected PFS. A marginal Cox regression model with clustering per study was used to assess differences in PFS between the subgroups. All statistical analyses were performed using R v3.4.4. (R Foundation for Statistical Computing, Vienna, Austria). This study was registered with PROSPERO (CRD42020125332).

\section{Results}

As shown in Table 1 and Figs. 1 and 2, 27 studies involving 23 different BCMA CAR-T-cell products were identified. Data were available from 640 BCMA CAR-T-cell treated patients. For 11 CAR-T-cell products, the extracellular BCMA-recognition domain of the CAR consisted of a human(ized) $\mathrm{mAb}$ in scFv format (Table 1) [55]. In one study (NCT03288493), the antigen-recognition domain was composed of a centyrin, a human fibronectin type III-based antibody mimetic [45, 56], while another (NCT03602612) used a human heavy-chain-only binding domain [44]. All other studies used non-human antibodies, either murine scFV mAb or nanobodies derived from alpaca or llama [46, 57]. Bb2121 and LCAR-B38M, the two most advanced BCMA CAR-T-cell products, used a murine- and llama antibody-based CAR construct, respectively (Table 2). The method used for T-cell enrichment/activation was not reported in the majority of the studies; anti-CD3 and anti-CD28 antibodies (usually coupled to magnetic beads) or an anti-CD3 antibody alone, with or without interleukin (IL)-2, were mostly used [58]. Lentiviral (489/640 patients; $76.4 \%)$ and, to a lesser extent, gamma-retroviral transduction (101/640 patients; $15.8 \%$ ) were the preferred transduction methods (Table 1 ). NCT03288493 (23/640 patients; $3.6 \%$ ) was the only clinical trial so far in which a non-viral delivery method was applied (i.e., a transposon). In two trials (ChiCTR-1800018143 and ChiCTR-1900027678), the method of CAR loading was not defined (Table 1) [33, 54]. In 520/640 patients (81.3\%), a 4-1BB-based secondgeneration CAR construct was used; the other patients received BCMA CAR-T cells with a CD28 co-stimulatory domain (either alone or in combination with OX40 or 4-1BB). One study (ChiCTR-1900027678) did not disclose the type of co-stimulatory domain [54]. CAR-T cell dosages varied considerably across the different studies, from $0.07 \times 10^{6} / \mathrm{kg}$ to $>1000 \times 10^{6}$ cells. This variation is also exemplified in Table 2, comparing bb2121 and LCAR-B38M, showing a tenfold difference between both studies in CAR-T-cell dosage used (Table 2). Cyclophosphamide, usually in combination with fludarabine, was the most frequently used lymphodepleting chemotherapy regimen.

Among 639 patients evaluable for safety, 80.3\% (69.0-88.2) experienced CRS (Table 1). CRS is graded on a scale from 1 to 4 [17]; severe CRS (i.e., grade $\geq 3$ ) occurred in $14.1 \%$ of patients (9.6-20.4). As shown in Table 2, detailing the key differences between the two 
Table 1 Multiple myeloma CAR-T-cell clinical trials targeting BCMA

\begin{tabular}{|c|c|c|c|c|c|c|c|c|c|}
\hline $\begin{array}{l}\text { Trial \# }{ }^{\text {ref. }} \text { (product } \\
\text { name) }\end{array}$ & $n=$ & $\begin{array}{l}\text { Origin } \\
\mathrm{mAb}\end{array}$ & Expansion & Loading & Co-stimulation & T-cell dosage & Conditioning & Toxicity & $\begin{array}{l}\text { Clinical } \\
\text { response }\end{array}$ \\
\hline $\begin{array}{l}\text { ChiCTR-OIC } 17011272 \\
\text { [27] (CD19 \& BCMA } \\
\text { CAR-T) }\end{array}$ & 21 & $\begin{array}{c}\text { Murine } \\
\text { scFv }\end{array}$ & $\mathrm{aCD} 3$ & Lentiviral & $4-1 B B$ & $1 \times 10^{6} / \mathrm{kg}$ & $\mathrm{CP} / \mathrm{Flu}$ & $\begin{array}{l}\text { CRS gr. 1-2 } \\
\quad(86 \%) \\
\text { gr. } \geq 3(5 \%) \\
\text { Neurotoxicity } \\
\quad(10 \%)\end{array}$ & $\begin{array}{l}\text { SCR/CR }(57 \%) / \\
\text { VGPR (24\%) } \\
\text { PR (14\%) }\end{array}$ \\
\hline $\begin{array}{l}\text { NCT02658929 }[24,28] \\
\quad(\text { bb2121) }\end{array}$ & $43 / 39$ & $\begin{array}{c}\text { Murine } \\
\text { scFv }\end{array}$ & $\mathrm{aCD} 3+\mathrm{aCD} 28$ & Lentiviral & $4-1 B B$ & $50-800 \times 10^{6}$ & $\mathrm{CP} / \mathrm{Flu}$ & $\begin{array}{l}\text { CRS gr. 1-2 } \\
\quad(58 \%) \\
\text { gr. } \geq 3(5 \%) \\
\text { Neurotoxicity } \\
(33 \%)\end{array}$ & $\begin{array}{l}\text { sCR/CR (44\%)/ } \\
\text { VGPR (23\%) } \\
\text { PR (10\%) }\end{array}$ \\
\hline $\begin{array}{l}\text { NCT03274219 [29] } \\
\quad(\text { bb21217) }\end{array}$ & 38 & $\begin{array}{c}\text { Murine } \\
\text { scFv }\end{array}$ & $\begin{array}{l}\mathrm{aCD} 3+\mathrm{aCD} 28 \\
+ \text { PI3k inhibitor }\end{array}$ & Lentiviral & $4-1 \mathrm{BB}$ & $150-450 \times 10^{6}$ & $\mathrm{CP} / \mathrm{Flu}$ & $\begin{array}{l}\text { CRS gr. } 1-2 \\
(61 \%) \\
\text { gr. } \geq 3(5 \%) \\
\text { Neurotoxicity } \\
(24 \%)\end{array}$ & $\begin{array}{l}\text { sCR/CR (13\%)/ } \\
\text { VGPR (34\%) } \\
\text { PR (5\%) }\end{array}$ \\
\hline $\begin{array}{l}\text { ChiCTR-OPC16009113 } \\
\quad[30,31](\text { BCMA- } \\
\text { CART) }\end{array}$ & 28 & $\begin{array}{l}\text { Murine } \\
\text { scFv }\end{array}$ & $\mathrm{aCD} 3$ & Lentiviral & CD28/4-1BB & $\begin{array}{l}5.4-25 \times 10^{6} / \\
\mathrm{kg}\end{array}$ & $\mathrm{CP} / \mathrm{Flu}$ & $\begin{array}{l}\text { CRS gr. } \geq 3 \\
\quad(14 \%)\end{array}$ & $\begin{array}{l}\text { sCR/CR (61\%)/ } \\
\text { VGPR (4\%) } \\
\text { PR (21\%) }\end{array}$ \\
\hline $\begin{array}{l}\text { NCT02215967 (1) } \\
{[25,32](\mathrm{NCI} B C M A} \\
\text { CAR-T) }\end{array}$ & 10 & $\begin{array}{l}\text { Murine } \\
\text { scFv }\end{array}$ & $\begin{array}{l}\mathrm{aCD} 3 \\
+\mathrm{IL}-2\end{array}$ & Retroviral & CD28 & $0.3-3 \times 10^{6} / \mathrm{kg}$ & $\mathrm{CP} / \mathrm{Flu}$ & $\begin{array}{l}\text { CRS gr. } 1-2 \\
(30 \%)\end{array}$ & $\begin{array}{l}\text { VGPR (10\%) } \\
\text { PR (10\%) }\end{array}$ \\
\hline $\begin{array}{l}\text { NCT02215967 (2) [25] } \\
\quad(\mathrm{NCI} \text { BCMA CAR-T) }\end{array}$ & 16 & $\begin{array}{l}\text { Murine } \\
\text { scFv }\end{array}$ & $\begin{array}{l}\mathrm{aCD} 3 \\
+\mathrm{IL}-2\end{array}$ & Retroviral & CD28 & $9 \times 10^{6} / \mathrm{kg}$ & CP/Flu & $\begin{array}{l}\text { CRS gr. 1-2 } \\
(56 \%) \\
\text { gr. } \geq 3 \\
(38 \%) \\
\text { Neurotoxicity } \\
(6 \%)\end{array}$ & $\begin{array}{l}\text { SCR/CR (13\%)/ } \\
\text { VGPR (50\%) } \\
\text { PR (19\%) }\end{array}$ \\
\hline $\begin{array}{l}\text { ChiCTR-1800018143 } \\
\text { [33] (BM38 CAR) }\end{array}$ & 22 & $\begin{array}{l}\text { Human- } \\
\text { ized } \\
\text { scFv }\end{array}$ & ND & ND & $4-1 \mathrm{BB}$ & $0.5-4 \times 10^{6} / \mathrm{kg}$ & $\mathrm{CP} / \mathrm{Flu}$ & $\begin{array}{l}\text { CRS gr. } 1-2 \\
(68 \%) \\
\text { gr. } \geq 3 \\
(23 \%)\end{array}$ & $\begin{array}{l}\text { sCR/CR (55\%)/ } \\
\text { VGPR (9\%) } \\
\text { PR (24\%) }\end{array}$ \\
\hline $\begin{array}{l}\text { NCT02546167 [20] } \\
\text { (CART-BCMA } \\
\text { UPenn) }\end{array}$ & 25 & $\begin{array}{l}\text { Human } \\
\text { scFv }\end{array}$ & aCD3/CD28 & Lentiviral & $4-1 \mathrm{BB}$ & $50-500 \times 10^{6}$ & CP or none & $\begin{array}{l}\text { CRS gr. 1-2 } \\
(56 \%) \\
\text { gr. } \geq 3 \\
(32 \%) \\
\text { Neurotoxicity } \\
(32 \%)\end{array}$ & $\begin{array}{l}\text { sCR/CR (8\%)/ } \\
\text { VGPR (20\%) } \\
\text { PR (20\%) }\end{array}$ \\
\hline $\begin{array}{l}\text { NCT03302403, } \\
\text { NCT03380039, } \\
\text { NCT03716856 [34, } \\
\text { 35] (CT053) }\end{array}$ & 24 & $\begin{array}{l}\text { Human } \\
\text { scFv }\end{array}$ & $\mathrm{aCD} 3 / \mathrm{CD} 28$ & Lentiviral & $4-1 \mathrm{BB}$ & $50-180 \times 10^{6}$ & $\mathrm{CP} / \mathrm{Flu}$ & $\begin{array}{l}\text { CRS gr. 1-2 } \\
(63 \%) \\
\text { Neurotoxicity } \\
\quad(8 \%)\end{array}$ & $\begin{array}{l}\text { sCR/CR (79\%)/ } \\
\text { VGPR (4\%) } \\
\text { PR (4\%) }\end{array}$ \\
\hline $\begin{array}{l}\text { NCT03430011 [36] } \\
\quad(J C A R H 125)\end{array}$ & 44 & $\begin{array}{l}\text { Human } \\
\text { scFv }\end{array}$ & ND & Lentiviral & $4-1 \mathrm{BB}$ & $50-450 \times 10^{6}$ & $\mathrm{CP} / \mathrm{Flu}$ & $\begin{array}{l}\text { CRS gr. 1-2 } \\
\quad(70 \%) \\
\text { gr. } \geq 3(9 \%) \\
\text { Neurotoxicity } \\
\quad(25 \%)\end{array}$ & $\begin{array}{l}\text { SCR/CR (27\%)/ } \\
\text { VGPR (20\%) } \\
\text { PR (34\%) }\end{array}$ \\
\hline $\begin{array}{l}\text { NCT03815383 [37] } \\
\quad(C-C A R 088)\end{array}$ & 5 & $\begin{array}{l}\text { Human } \\
\text { scFv }\end{array}$ & ND & Lentiviral & $4-1 \mathrm{BB}$ & $1-3 \times 10^{6} / \mathrm{kg}$ & $\mathrm{CP} / \mathrm{Flu}$ & $\begin{array}{l}\text { CRS gr. 1-2 } \\
(80 \%)\end{array}$ & $\begin{array}{l}\text { sCR/CR (20\%)/ } \\
\text { VGPR (60\%) } \\
\text { PR (20\%) }\end{array}$ \\
\hline $\begin{array}{l}\text { ChiCTR-1800018137 } \\
\text { [38] (CT103A) }\end{array}$ & 18 & $\begin{array}{l}\text { Human } \\
\text { scFv }\end{array}$ & ND & Lentiviral & $4-1 \mathrm{BB}$ & $1-6 \times 10^{6} / \mathrm{kg}$ & $\mathrm{CP} / \mathrm{Flu}$ & $\begin{array}{c}\text { CRS gr. } 1-2 \\
(72 \%) \\
\text { gr. } \geq 3 \\
(22 \%)\end{array}$ & $\begin{array}{l}\text { sCR/CR (67\%)/ } \\
\text { VGPR (17\%) } \\
\text { PR (17\%) }\end{array}$ \\
\hline $\begin{array}{l}\text { NCT03549442 } \\
\text { [39] (CART- } \\
\text { BCMA + CTL119) }\end{array}$ & 16 & $\begin{array}{l}\text { Human } \\
\text { scFv }\end{array}$ & ND & Lentiviral & $4-1 \mathrm{BB}$ & $500 \times 10^{6}$ & $\mathrm{CP} / \mathrm{Flu}$ & $\begin{array}{l}\text { CRS gr. 1-2 } \\
(88 \%)\end{array}$ & $\begin{array}{l}\text { SCR/CR (19\%)/ } \\
\text { VGPR (25\%) } \\
\text { PR (25\%) }\end{array}$ \\
\hline $\begin{array}{l}\text { NCT03338972 [40] } \\
\quad \text { (FCARH143) }\end{array}$ & 11 & $\begin{array}{l}\text { Human } \\
\text { scFv }\end{array}$ & $\mathrm{aCD} 3 / \mathrm{CD} 28$ & Lentiviral & $\begin{array}{l}\text { 4-1BB } \\
+ \text { EGFRt }\end{array}$ & $50-150 \times 10^{6}$ & $\mathrm{CP} / \mathrm{Flu}$ & $\begin{array}{l}\text { CRS gr. 1-2 } \\
\text { (91\%) } \\
\text { Neurotoxicity } \\
(9 \%)\end{array}$ & $\begin{array}{l}\text { SCR/CR (55\%)/ } \\
\text { VGPR (36\%) } \\
\text { PR (9\%) }\end{array}$ \\
\hline
\end{tabular}


Table 1 (continued)

\begin{tabular}{|c|c|c|c|c|c|c|c|c|c|}
\hline $\begin{array}{l}\text { Trial \# }{ }^{\text {ref. }} \text { (product } \\
\text { name) }\end{array}$ & $n=$ & $\begin{array}{l}\text { Origin } \\
\mathrm{mAb}\end{array}$ & Expansion & Loading & Co-stimulation & T-cell dosage & Conditioning & Toxicity & $\begin{array}{l}\text { Clinical } \\
\text { response }\end{array}$ \\
\hline $\begin{array}{l}\text { NCT03502577 [41] } \\
\quad(\text { FCARH143 + GSI) }\end{array}$ & 10 & $\begin{array}{l}\text { Human } \\
\text { scFv }\end{array}$ & ND & Lentiviral & $\begin{array}{l}\text { 4-1BB } \\
+ \text { EGFRt }\end{array}$ & $50-300 \times 10^{6}$ & $\mathrm{CP} / \mathrm{Flu}$ & $\begin{array}{c}\text { CRS gr. 1-2 } \\
(60 \%) \\
\text { gr. } \geq 3 \\
(40 \%) \\
\text { Neurotoxicity } \\
(60 \%)\end{array}$ & $\begin{array}{l}\text { sCR/CR (30\%)/ } \\
\text { VGPR (50\%) } \\
\text { PR (20\%) }\end{array}$ \\
\hline $\begin{array}{l}\text { NCT03196414 [42] } \\
\text { (SZ-MM-CART01) }\end{array}$ & $29 / 28$ & $\begin{array}{l}\text { Human- } \\
\text { ized } \\
\text { scFv }\end{array}$ & $\mathrm{aCD} 3$ & Lentiviral & CD28/OX40 & $20-82 \times 10^{6} / \mathrm{kg}$ & CP/Flu & $\begin{array}{l}\text { CRS gr. 1-2 } \\
(66 \%) \\
\text { gr. } \geq 3 \\
(34 \%) \\
\text { Neurotoxicity } \\
(3 \%)\end{array}$ & $\begin{array}{l}\text { SCR/CR (54\%)/ } \\
\text { VGPR (4\%) } \\
\text { PR (29\%) }\end{array}$ \\
\hline $\begin{array}{l}\text { NCT03455972 [43] } \\
\text { (SZ-MM-CART02) }\end{array}$ & 32 & $\begin{array}{l}\text { Human- } \\
\text { ized } \\
\text { scFv }\end{array}$ & $\mathrm{aCD} 3$ & Lentiviral & CD28/OX40 & $50 \times 10^{6} / \mathrm{kg}$ & $\begin{array}{l}\text { BUCY or Mel } \\
\text { + autoHSCT }\end{array}$ & $\begin{array}{l}\text { CRS gr. } 1-2 \\
\quad(97 \%) \\
\text { gr. } \geq 3(3 \%)\end{array}$ & $\begin{array}{l}\text { sCR/CR (72\%)/ } \\
\text { VGPR (ND) } \\
\text { PR (ND) }\end{array}$ \\
\hline $\begin{array}{l}\text { NCT03070327 [26] } \\
\text { (MCARH171) }\end{array}$ & $10 / 11$ & $\begin{array}{l}\text { Human } \\
\text { scFv }\end{array}$ & ND & Retroviral & $\begin{array}{l}\text { 4-1BB } \\
+\mathrm{EGFRt}\end{array}$ & $\begin{array}{l}1 \times 10^{6} / \mathrm{kg} \text { or } \\
150-450 \times 10^{6}\end{array}$ & $\mathrm{CP} /$ Flu or $\mathrm{CP}$ & $\begin{array}{l}\text { CRS gr. 1-2 } \\
(40 \%) \\
\text { gr. } \geq 3 \\
(20 \%) \\
\text { Neurotoxicity } \\
(10 \%)\end{array}$ & $\begin{array}{l}\text { VGPR (45\%) } \\
\text { PR (18\%) }\end{array}$ \\
\hline $\begin{array}{l}\text { NCT03602612 [44] } \\
\text { (FHVH33) }\end{array}$ & 15 & $\begin{array}{l}\text { Human } \\
\text { VH }\end{array}$ & ND & Retroviral & $4-1 B B$ & ND & $\mathrm{CP} / \mathrm{Flu}$ & $\begin{array}{l}\text { CRS gr. 1-2 } \\
(87 \%), \\
\text { gr. } \geq 3(7 \%) \\
\text { Neurotoxicity } \\
(27 \%)\end{array}$ & $\begin{array}{l}\text { sCR/CR (20\%)/ } \\
\text { VGPR (7\%) } \\
\text { PR (53\%) }\end{array}$ \\
\hline $\begin{array}{l}\text { NCT03288493 [45] } \\
\quad(P-B C M A-101)\end{array}$ & $23 / 19$ & $\begin{array}{l}\text { Human } \\
\text { cen- } \\
\text { tyrin }\end{array}$ & None & $\begin{array}{l}\text { Transpo- } \\
\text { son }\end{array}$ & $\begin{array}{l}\text { 4-1BB } \\
\text { + rimiducid SS }\end{array}$ & $51-1143 \times 10^{6}$ & CP/Flu & $\begin{array}{l}\text { CRS gr. 1-2 } \\
(9 \%) \\
\text { Neurotoxicity } \\
(4 \%)\end{array}$ & $\begin{array}{l}\mathrm{SCR} / \mathrm{CR}+\mathrm{VGPR} \\
(26 \%) \\
\operatorname{PR}(42 \%)\end{array}$ \\
\hline $\begin{array}{l}\text { NCT03661554 [46] } \\
\text { (BCMA nanoanti- } \\
\text { body) }\end{array}$ & 9 & $\begin{array}{c}\text { Alpaca } \\
\mathrm{V}_{\mathrm{H}} \mathrm{H}\end{array}$ & ND & Lentiviral & $4-1 B B$ & $250-900 \times 10^{6}$ & $\mathrm{CP} /$ Flu & $\begin{array}{l}\text { CRS gr. 1-2 } \\
(67 \%) \\
\text { gr. } \geq 3 \\
(22 \%) \\
\text { Neurotoxicity } \\
(11 \%)\end{array}$ & $\begin{array}{l}\text { SCR/CR (56\%)/ } \\
\text { VGPR (33\%) } \\
\text { PR (11\%) }\end{array}$ \\
\hline $\begin{array}{c}\text { NCT03090659 (1) [47, } \\
\text { 48] (LCAR-B38M) }\end{array}$ & 17 & $\begin{array}{r}\text { Llama } \\
\qquad \mathrm{V}_{\mathrm{H}} \mathrm{H}\end{array}$ & $\begin{array}{l}\mathrm{aCD} 3 / \mathrm{CD} 28 \\
+\mathrm{IL}-2\end{array}$ & Lentiviral & 4-1BB & $\begin{array}{l}0.21- \\
1,52 \times 10^{6} \% \\
\mathrm{~kg}\end{array}$ & $\mathrm{CP} /$ Flu or $\mathrm{CP}$ & $\begin{array}{l}\text { CRS gr. 1-2 } \\
(59 \%) \\
\text { gr. } \geq 3 \\
(41 \%)\end{array}$ & $\begin{array}{l}\text { sCR/CR }(82 \%) / \\
\operatorname{VGPR}(6 \%)\end{array}$ \\
\hline $\begin{array}{l}\text { NCT03090659 (2) [49, } \\
\text { 50] (LCAR-B38M) }\end{array}$ & 57 & $\begin{array}{r}\text { Llama } \\
\mathrm{V}_{\mathrm{H}} \mathrm{H}\end{array}$ & $\begin{array}{l}\mathrm{aCD} 3 / \mathrm{CD} 28 \\
+\mathrm{IL}-2\end{array}$ & Lentiviral & 4-1BB & $\begin{array}{l}0.07-2,1 \times 10^{6} \% \\
\mathrm{~kg}\end{array}$ & $\mathrm{CP}$ & $\begin{array}{l}\text { CRS gr. 1-2 } \\
(82 \%) \\
\text { gr. } \geq 3(7 \%) \\
\text { Neurotoxicity } \\
(2 \%)\end{array}$ & $\begin{array}{l}\text { SCR/CR (73\%)/ } \\
\text { VGPR (4\%) } \\
\text { PR (11\%) }\end{array}$ \\
\hline $\begin{array}{l}\text { NCT03548207 [51] } \\
\quad(\text { LCAR-B38M) }\end{array}$ & 29 & $\begin{array}{r}\text { Llama } \\
\mathrm{V}_{\mathrm{H}} \mathrm{H}\end{array}$ & ND & Lentiviral & 4-1BB & $\begin{array}{l}0.5-0.9 \times 10^{6} / \\
\mathrm{kg}\end{array}$ & $\mathrm{CP} /$ Flu & $\begin{array}{l}\text { CRS gr. 1-2 } \\
(86 \%) \\
\text { gr. } \geq 3(7 \%) \\
\text { Neurotoxicity } \\
(10 \%)\end{array}$ & $\begin{array}{l}\text { SCR/CR (69\%)/ } \\
\text { VGPR (17\%) } \\
\text { PR (14\%) }\end{array}$ \\
\hline $\begin{array}{r}\text { ChiCTR-1800017404 } \\
\text { [52] (BCMA CAR-T) }\end{array}$ & $33 / 32$ & ND & ND & Lentiviral & 4-1BB & $1-6 \times 10^{6} / \mathrm{kg}$ & $\mathrm{CP} / \mathrm{Flu}$ & $\begin{array}{l}\text { CRS gr. } 1-2 \\
(52 \%) \\
\text { gr. } \geq 3 \\
(48 \%)\end{array}$ & $\begin{array}{l}\text { SCR/CR (66\%)/ } \\
\text { VGPR (22\%) } \\
\text { PR (13\%) }\end{array}$ \\
\hline $\begin{array}{l}\text { NCT03093168 [53] } \\
\text { (HRAIN BCMA- } \\
\text { CART) }\end{array}$ & 49 & ND & ND & Retroviral & $\begin{array}{l}\text { 4-1BB } \\
+ \text { EGFRt }\end{array}$ & $9 \times 10^{6} / \mathrm{kg}$ & CP/Flu & $\begin{array}{l}\text { CRS gr. } 1-2 \\
\quad(12 \%) \\
\text { gr. } \geq 3(6 \%)\end{array}$ & $\begin{array}{l}\text { SCR/CR (45\%)/ } \\
\text { VGPR (18\%) } \\
\text { PR (14\%) }\end{array}$ \\
\hline $\begin{array}{l}\text { ChiCTR-1900027678 } \\
\text { [54] (GC012F) }\end{array}$ & 5 & ND & ND & ND & ND & $1-2 \times 10^{6} / \mathrm{kg}$ & $\mathrm{CP} /$ Flu or none & $\begin{array}{l}\text { CRS gr. 1-2 } \\
(80 \%)\end{array}$ & $\begin{array}{c}\text { sCR/CR (20\%)/ } \\
\text { VGPR (80\%) }\end{array}$ \\
\hline \multirow[t]{3}{*}{ Pooled studies } & $639 / 630$ & & & & & & & $\begin{array}{c}\text { CRS gr. } 1-4 \\
(80.3 \%)\end{array}$ & ORR $(80.5 \%)$ \\
\hline & & & & & & & & $(95 \% \mathrm{Cl}$ & $(95 \% \mathrm{Cl}$ \\
\hline & & & & & & & & $\begin{array}{l}\text { 69.0-88.2; } \\
\left.I^{2}=83 \%\right)\end{array}$ & $\begin{array}{l}73.5-85.9 \\
\left.I^{2}=61 \%\right)\end{array}$ \\
\hline
\end{tabular}


Table 1 (continued)

\begin{tabular}{|c|c|c|c|c|c|c|c|c|c|}
\hline $\begin{array}{l}\text { Trial \# }{ }^{\text {ref. }} \text { (product } \\
\text { name) }\end{array}$ & $n=$ & $\begin{array}{l}\text { Origin } \\
\mathrm{mAb}\end{array}$ & Expansion & Loading & Co-stimulation & T-cell dosage & Conditioning & Toxicity & $\begin{array}{l}\text { Clinical } \\
\text { response }\end{array}$ \\
\hline & & & & & & & & $\begin{array}{l}\text { Neurotoxic- } \\
\text { ity }(10.5 \%)\end{array}$ & \\
\hline & & & & & & & & $\begin{array}{l}(95 \% \mathrm{Cl} \\
6.8-16.0 \\
\left.I^{2}=58 \%\right)\end{array}$ & \\
\hline
\end{tabular}

$\mathrm{aCD} 3+\mathrm{aCD} 28=$ anti-CD3 and anti-CD28 antibodies. $\mathrm{aCD} 3 / \mathrm{CD} 28+\mathrm{IL}-2=$ anti-CD3 and anti-CD28-coated beads plus interleukin-2. AutoHSCT $=$ autologous hematopoietic stem cell transplant. BCMA = B-cell maturation antigen. BUCY = busulfan and cyclophosphamide. $\mathrm{CAR}=\mathrm{chimeric}$ antigen receptor. $\mathrm{CP}=$ cyclophosphamide. $\mathrm{CR}=$ complete response. $\mathrm{CRS}=$ cytokine release syndrome. $\mathrm{EGFRt}=$ truncated epidermal growth factor receptor. Flu $=$ fludarabine. Gr. = grade. GSI= gamma-secretase inhibitor. IL-2 = interleukin-2. Mel=melphalan. $\mathrm{n}=$ number of patients evaluable for toxicity/clinical response. ND=not disclosed. $\mathrm{PI} 3 \mathrm{k}=$ phosphoinositide 3-kinase. $\mathrm{PR}=$ partial response. $\mathrm{scFv}=$ single-chain fragment variable. $\mathrm{SS}=$ safety switch. $\mathrm{sCR}=$ stringent complete response. Trial \#=study registration number in Clinicaltrials.gov (NCT\#) or Chinese Clinical Trial Registry (ChiCTR-\#). VGPR $=$ very good partial response. $\mathrm{V}_{\mathrm{H}} \mathrm{H}=$ nanobody

most advanced BCMA CAR-T products bb2121 and LCAR-B38M, the median time of CRS onset varied greatly between 1 and 9 days. The median duration was between 7 and 9 days for bb2121 and LCAR-B38M, respectively; CRS could last to up to 2 months (Table 2). The pooled CRS rate was $61.0 \%\left(35.3-81.8 ; I^{2}=84 \%\right)$, 83.8\% (70.9-91.7; $\left.I^{2}=71 \%\right)$, and $91.0 \% \quad(83.8-95.2$; $\left.I^{2}=0 \%\right)$ in studies using CAR constructs with murinebased, human(ized), and alpaca/llama-derived antigen-binding domains, respectively (Additional file 2: Table S2 and Fig. S1). Despite the apparently lower CRS rate in studies using murine scFv-based CAR constructs, individual studies revealed a clear "dosetoxicity" relation. For example, with the bb2121 CAR-T product, which contains a murine anti-BCMA scFv, a CRS rate of $96.3 \%$ was noted at the recommended phase II dose of $450 \times 10^{6}$ cells (Table 2), whereas it was only $75.7 \%$ and $50.0 \%$ at the $300 \times 10^{6}$ and $150 \times 10^{6}$ dose levels, respectively [59].

The pooled neurotoxicity rate was $10.5 \%(6.8-16.0)$, with a considerable variation between the different studies. For example, in the bb2121 study, 20.4\% of the patient experienced some sort of neurological symptoms, whereas only $1.8 \%$ of the LCAR-B38M-treated patients had neurotoxicity (Table 2). The origin of the antigen-recognition domain (murine, human(ized), or alpaca/llama) had no impact on neurotoxicity (Additional file 3: Table S3). Lymphodepletion with cyclophosphamide and fludarabine, a known neurotoxic agent, did not lead to more neurological events as compared to cyclophosphamide alone or no lymphodepletion. A lower rate of neurotoxicity was observed in studies that used anti-CD3 mAbs alone instead of anti-CD3/CD28 mAbs for T-cell enrichment/activation $\left(4.9 \%\right.$ [2.1-10.9; $I^{2}=0 \%$ ] versus $15.9 \%$ [8.1-28.9; $\left.\left.I^{2}=66 \%\right] ; p=0.028\right)$. A similar observation was made for studies that used CD28 instead of $4-1 \mathrm{BB}$ as costimulatory backbone $\left(3.4 \%\left[1.2-9.3 ; I^{2}=0 \%\right]\right.$ versus $\left.12.9 \%\left[8.2-19.6 ; I^{2}=59 \%\right] ; p=0.018\right)$. A higher rate of neurotoxicity was observed in studies in which the median patient age was $\geq 60$ years $(20.5 \%$ [12.5-31.9; $\left.I^{2}=63 \%\right]$ versus $\left.6.4 \%\left[3.3-12.0 ; I^{2}=38 \%\right] ; p=0.0043\right)$, and in studies in which the median number of prior lines of therapy was $\geq 5\left(19.1 \%\left[13.3-26.7 ; I^{2}=45 \%\right]\right.$ versus $2.8 \%\left[1.3-6.1 ; I^{2}=0 \%\right] ; p<0.0001 ;$ Additional file 3: Fig. S2).

A total of 630 patients were evaluable for clinical response (Table 1). The pooled ORR was $80.5 \%$ (73.5-85.9) with (s)CR in 44.8\% (35.3-54.6) of patients. Responses occurred rapidly, usually within the first month after CAR-T-cell infusion. Despite the higher likelihood to achieve a deep response in studies that included less pretreated patients (CR: 57.6\% [45.2-69.0; $\left.I^{2}=63 \%\right]$; $p=0.011)$, a (s)CR rate of $32.9 \%\left(21.1-47.4 ; I^{2}=77 \%\right)$ was still achieved in studies with a median of $\geq 5$ prior lines of therapy. Concerning the treatment-related variables, a superior CR rate of $71.9 \%\left(62.8-79.6 ; I^{2}=0\right)$ was noted in studies with an alpaca/llama-derived BCMA-recognition domain $(p<0.0001$ compared to their human and murine counterparts; Additional file 4: Fig. S3). Responses were usually deeper in studies that used an alpaca/llamabased anti-BCMA CAR construct, as exemplified by LCAR-B38M in Table 2. Finally, the CR rate was only $18.0 \%\left(6.5-41.1 ; I^{2}=67 \%\right)$ in studies that used a retroviral instead of a lentiviral vector $\left(50.6 \%\right.$ [39.8-61.4; $\left.\left.I^{2}=77 \%\right]\right)$ for CAR-T-cell transduction $(p=0.015$; Additional file 4 : Table S4).

PFS data were available for 551 patients; the median PFS of patients treated with active BCMA CAR-T-cell doses was 12.2 months (11.4-17.4), comparing favorably to the 1.9-month PFS (1.5-3.7) observed in patients treated with inactive doses in the dose-escalation studies NCT02658929, NCT02546167, NCT02215967, and NCT03070327 (hazard ratio [HR] 0.14; $p<0.0001$; Fig. 3a). In line with the superior clinical response rate, patients treated with lentivirally transduced CAR-T cells had a significantly longer PFS than those treated with retroviral constructs (12.8 months [11.4-19.9] 
Table 2 Comparison of KarMMa (bb2121) and LEGEND-2 (LCAR-B38M) clinical studies

\begin{tabular}{|c|c|c|}
\hline & bb2121 / KarMMa [59] & $\begin{array}{l}\text { LCAR-B38M / LEGEND-2 } \\
\text { (Xi'an site) }[49,50]\end{array}$ \\
\hline Alternative product name & ide-cel & cilta-cel \\
\hline Trial \# (study phase) & NCT03361748 (phase II) & NCT03090659 (phase I) \\
\hline$n$ of patients & $128\left(54\right.$ at RD of $\left.450 \times 10^{6}\right)$ & 57 \\
\hline Expansion method & $\mathrm{aCD} 3+\mathrm{aCD} 28$ & $\mathrm{aCD} 3 / \mathrm{CD} 28+\mathrm{IL}-2$ \\
\hline Loading method & Lentiviral & Lentiviral \\
\hline CAR-T structure & 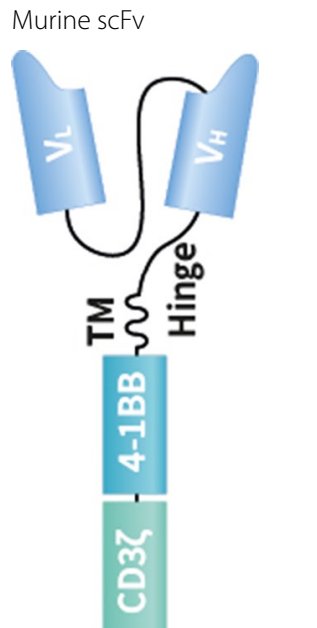 & Llama $2 \times \mathrm{V}_{\mathrm{H}} \mathrm{H}$ \\
\hline Lymphodepletion & CP/Flu & $C P$ \\
\hline CAR-T cell dosage(s) & $150-300$ to $450 \times 10^{6}$ & $32.3 \times 10^{6}\left(3.3\right.$ to $\left.126.2 \times 10^{6}\right)$ \\
\hline \multicolumn{3}{|l|}{ Patient characteristics } \\
\hline Age (range), y & $61(33-78)$ & $54(27-72)$ \\
\hline Median n PLT (range) & $6(3-16)$ & $3(1-9)$ \\
\hline High-risk features $^{a}$ & $51 \%$ & $37 \%$ \\
\hline CRS & $96.3 \%^{b}$ & $89.5 \%$ \\
\hline Gr. 1-2 & $90.7 \%$ & $82.5 \%$ \\
\hline $\mathrm{Gr} . \geq 3$ & $5.6 \%$ & $7.0 \%$ \\
\hline Median onset (range) & $1 d(1-10)$ & $9 d(1-19)$ \\
\hline Median duration (range) & $7 d(1-63)$ & $9 d(3-57)$ \\
\hline Tocilizumab use & $67 \%$ & $46 \%$ \\
\hline Neurotoxicity & $20.4 \%^{b}$ & $1.8 \%$ \\
\hline ORR & $82 \%^{b}$ & $88 \%$ \\
\hline $\mathrm{MRD}^{-} \mathrm{CR}$ & $28 \%$ & $68 \%$ \\
\hline$C R$ & $11 \%$ & $5 \%$ \\
\hline VGPR & $26 \%$ & $4 \%$ \\
\hline PR & $17 \%$ & $11 \%$ \\
\hline Median PFS (95\% Cl) & $12.1 \mathrm{~m}(8.8-12.3)^{\mathrm{b}}$ & $19.9 m(9.6-31)$ \\
\hline
\end{tabular}

$\mathrm{aCD} 3+\mathrm{aCD} 28=$ anti-CD3 and anti-CD28 antibodies. $\mathrm{aCD} 3 / \mathrm{CD} 28+\mathrm{IL}-2=$ anti-CD3 and anti-CD28-coated beads plus interleukin-2. cilta-cel $=$ ciltacabtagene autoleucel. $\mathrm{CP}=$ cyclophosphamide. $\mathrm{CP} / \mathrm{Flu}=$ cyclophosphamide plus fludarabine. $\mathrm{CR}=$ complete response. $\mathrm{CRS}=\mathrm{cytokine}$ release syndrome. $\mathrm{d}=\mathrm{days}$. Gr. $=$ grade. ide-cel $=$ idecabtagene vicleucel. $m=$ months. $M R D=$ minimal residual disease. $n=$ number. $O R R=$ objective response rate. $P F S=$ progression-free survival. $P L T=$ prior lines of treatment. $\mathrm{RD}=$ recommended dose. $\mathrm{scFv}=$ single-chain variable fragment. (VG)PR= (very good) partial response. $\mathrm{VHH}=\mathrm{heavy}$-chain variable region. Trial $\#=$ study registration number in Clinicaltrials.gov (NCT\#). $y=$ years

a High-risk defined as R-ISS stage 3 and/or high-risk genetics (del(17p), t(4;14), t(14;16))

b Data shown for the $450 \times 10^{6}$ dose cohort only 


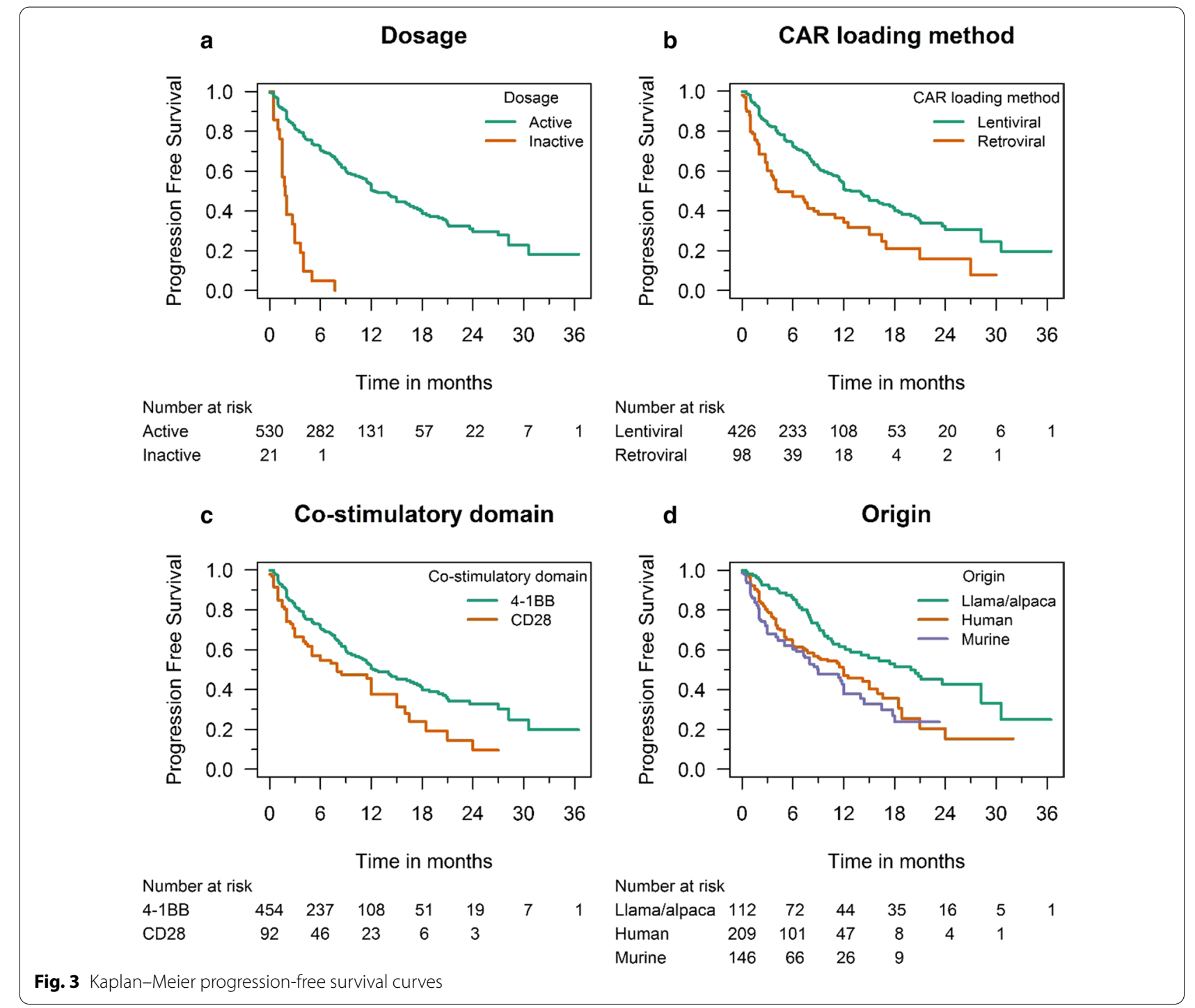

versus 4.3 months [3.0-15.0]; HR 0.48; $p=0.0065$; Fig. 3b; Additional file 4: Table S4). Although no difference was seen in terms of ORR, we observed a shorter PFS among patients treated with BCMA CAR-T-cells containing a CD28-based co-stimulatory backbone (8.0 months [4.0-15.0] versus 12.2 months [10.8-17.4] with a 4-1BB-based co-stimulatory domain); however, this difference was not statistically significant (HR 0.63, $p=0.061$; Fig. 3c). The median PFS in the bb2121 study was 12.1 months (8.8-12.3); in the LCAR-B38M study, a median PFS of 19.9 months (9.6-31) was reported (Table 2). The longest PFS rates were observed in studies that used alpaca/llama constructs $(p=0.0005$; Fig. 3d; Additional file 2: Table S2).

\section{Discussion}

This meta-analysis provides insights into the risk and benefits of BCMA CAR-T-cell therapy in MM, into the diversity of the patient populations included and BCMA CAR-T-cell products used, and into the various factors that potentially contribute to toxicity and efficacy. As of January 1, 2020, 27 registered clinical studies have been published involving 23 different CAR-T-cell products and 640 patients. A high response rate was observed, with demonstrable responses in $8 / 10$ patients (nearly half of whom had a CR). Toxicity was equally high, with CRS occurring in $8 / 10$ patients and neurotoxicity in $1 / 10$ patients. Despite the high initial response rate, responses were usually temporary and relapses were frequently observed, resulting in a 
median PFS of 12.2 months in patients receiving active doses of BCMA CAR-T cells. The two most advanced BCMA CAR-T products are bb2121 or idecabtagene vicleucel (which contains a murine anti-BCMA scFv as antigen-recognition domain) and LCAR-B38M or ciltacabtagene autoleucel (which contains two llama antiBCMA heavy chain variable regions or $\mathrm{V}_{\mathrm{H}} \mathrm{H}$ ) [24, 48, 50].

At the recommended phase II dose level of $450 \times 10^{6}$ cells, the murine BCMA CAR-T product bb2121 yielded comparable CRS rates as its human(ized) or alpaca/ llama-based counterparts (Table 2) [59], indicating that not the species origin of the CAR antigen-recognition domain but the CAR-T cell dosage is a major determinant of CRS. Albeit mostly low grade, neurotoxicity occurred in up to 1 out of 5 patients treated with bb2121; in LCARB38M-treated patients, the neurotoxicity rate was tenfold lower (Table 2). Since the origin of the antigen-recognition domain (murine, human, or alpaca/llama) was not found to be a risk factor for neurotoxicity in this metaanalysis, other factors should have contributed to the observed difference in neurotoxicity rate between bb2121 and LCAR-B38M. In the LEGEND-2 study conducted at the Xi'An site in China (NCT03090659) [49, 50], the largest study with LCAR-B38M published to date, the lymphodepleting regimen consisted of cyclophosphamide alone, whereas cyclophosphamide and fludarabine were used in the KarMMa pivotal phase II study with bb2121 (NCT03361748) (Table 2). The use of fludarabine for lymphodepletion, which by itself can cause neurotoxicity, was shown to increase the risk of neurologic adverse events in the CD19 CAR-T-cell field [60]. Our meta-analysis, however, failed to demonstrate a role for fludarabine in the higher rate of neurotoxicity in the KarMMa study of bb2121. Dual anti-CD3/CD28 stimulation during CAR-T-cell culture and 4-1BB as co-stimulatory domain were identified as potential risk factors for neurotoxicity in this study. As indicated in Table 2, both KarMMa (bb2121) and LEGEND-2 (LCAR-B38M) used anti-CD3/CD28-stimulated 4-1BB-based CAR-T cells. We, therefore, believe that these factors are not major drivers of neurotoxicity and, at least, do not explain the difference in neurotoxicity rates between both studies. Although this is in sharp contrast with what has been observed in studies with CD19 CAR-T cells [60, 61], this meta-analysis pointed to a higher risk of neurotoxicity in BCMA CAR-T-cell studies in which the median patient age was $\geq 60$ years and/or in which the median number of prior lines of anti-myeloma treatments was $\geq 5$. As shown in Table 2, LEGEND-2 (LCAR-B38M) tended to include younger and less pretreated patients, possibly explaining the lower frequency of neurological events as compared to KarMMa (bb2121).
Although a previous clinical trial of CD19 CAR-T-cell therapy in CLL failed to demonstrate such correlation [62], we observed a lower rate of deep responses ([s]CR) in studies that included more heavily pretreated patients ( $\geq 5$ prior lines of treatment). This explains why in the LEGEND-2 study a higher proportion of LCAR-B38Mtreated patients achieved an (MRD-negative) CR status as compared to the bb2121-treated patients in KarMMA. The rationale behind this is that apheresis products of less pretreated MM patients contain "fitter" $\mathrm{T}$ cells [63], resulting in better clinical responses. Autologous BCMA CAR-T-cell therapies are now being positioned earlier in the course of the disease (NCT03549442, NCT03455972) in an attempt to produce deeper and more durable clinical responses. The fact that KarMMa included more high-risk MM patients as compared to LEGEND-2 (Table 2) likely played no role in the lower deep response rate. Indeed, in this meta-analysis, myeloma risk was not associated with reduced activity, indicating that BCMA CAR-T-cell therapy is also highly efficacious in the high need subgroup of high-risk MM patients. Another factor possibly contributing to the superior therapeutic activity of LCAR-B38M is related to the use of (two) llama $\mathrm{V}_{\mathrm{H}} \mathrm{Hs}$ as antigen-binding domain in contrast to the murine scFv-based CAR construct of bb2121. It is known that CARs based on heavy-chain-only antibodies (such as alpaca or llama-derived $\mathrm{V}_{\mathrm{H}} \mathrm{H}$ ) have superior BCMA-binding capability of $\mathrm{V}_{\mathrm{H}} \mathrm{H}$ compared to traditional scFv-based domains $[64,65]$. This is also reflected by the fact that tenfold lower CAR-T cell dosages were required in LEGEND-2 (LCAR-B38M) as compared to KarMMa (bb2121). To summarize, although head-tohead trials between bb2121 and LCAR-B38M have not been conducted, the results of this meta-analysis indicate that the differences in terms of MRD-negativity, depth of response, and, consequently, PFS, between both products are in large part attributable to the different patient populations included and possibly also to the type of antigenrecognition domain used.

Although there was no statistically significant difference in terms of ORR, PFS was markedly longer in the 4-1BB subgroup. This corroborates recent research showing longer CAR-T-cell persistence and improved response durability with 4-1BB-based as compared to CD28-based CD19 CAR-T cells [66]. Although this should still be confirmed in a randomized controlled trial, our results also seem to favor the use of lentiviral over retroviral vectors for CAR-T-cell transduction given their superior clinical activity without increasing toxicity. Non-viral CAR loading methods, such as DNA transposons, are gaining popularity but how these compare to lentiviral or retroviral transduction in terms of toxicity and activity remains to be established. 
We observed a sixfold increase in median PFS in the treatment group compared to the control group, which received an inactive CAR-T-cell dose. The low PFS ( $\sim 2$ months $)$ in the control group is congruent with previous literature [67] and illustrates the grim prognosis of the patients included so far in BCMA CAR-Tcell studies. In contrast to what is observed in the field of CD19-directed CAR-T-cell therapy for diffuse large B-cell lymphoma, the tail of the PFS curve did not reveal a plateau. This indicates that the majority of the patients will eventually relapse. Possible explanations are lack of CAR-T cell persistence, antigen escape, the hostile tumor microenvironment, and exhaustion. Persistence can be improved by altering the CD4/CD8 composition of the infusion product $[21,40]$, or by enriching the product with stem cell memory $\mathrm{T}$ cells $[45,68]$. BCMA downregulation or loss was observed in several trials [20, 25, $32,40]$; this can be mediated by shedding of BCMA from the cell surface [8] or by CAR-T cell-induced trogocytosis. The latter not only leads to reduced tumor cell recognition, but also to CAR-T-cell fratricide [69]. In order to prevent BCMA shedding, $\gamma$-secretase inhibitors are being combined with BCMA CAR-T cells (NCT03502577) [41]. Another approach to circumvent antigen escape is cotargeting of BCMA and another antigen, such as CD19 (NCT03196414, NCT03455972) [27, 43], or simultaneous targeting of two BCMA epitopes as in LCAR-B38M [47-51]. Relapse can also occur despite CAR-T-cell persistence and maintained BCMA expression. The hypoxic niche in the BM, where MM cells reside, impairs cytokine secretion and granzyme $\mathrm{B}$ release from BCMA CAR-T cells [70]. In addition, upregulation of immune checkpoint molecules, such as programmed death-1 (PD-1), results in BCMA CAR-T-cell exhaustion which can be restored by PD-1 blockade [71]. Tonic signaling in the absence of antigen can induce CAR-T-cell exhaustion as well; proper selection of the antigen-recognition domain [45] and the co-stimulatory domain [69] can help to minimize CAR tonic signaling.

In conclusion, this meta-analysis provides robust evidence for the high clinical activity of BCMA CAR-T-cell therapies in $\mathrm{MM}$ and shows that several patient- and treatment-related factors might contribute to their toxicity and efficacy. These findings may inform the design of future CAR-T-cell studies in MM.

\section{Supplementary information}

Supplementary information accompanies this paper at https://doi. org/10.1186/s13045-020-01001-1.

Additional file 1. Overview of the 61 publications identified following the PRISMA flow diagram.
Additional file 2. Subgroup comparison for antigen-recognition domain origin and forest plot for CRS (grouped by antigen-recognition domain).

Additional file 3. Subgroup comparison for neurotoxicity and forest plot for neurotoxicity (grouped by lines of prior therapy).

Additional file 4. Subgroup comparison for CAR loading method and forest plot for (s)CR (grouped by antigen-recognition domain).

\section{Acknowledgements}

Part of this work was presented during the 4th French International Symposium on CART Cells (CART Day), which took place in Lille, France, from 15/01/2020 until 16/01/2020, and which was organized by Prof Suman Mitra and Prof Ibrahim Yakoub-Agha (Lille, France).

\section{Authors' contributions}

SA conceived the study design; SA, MT, and GR did the literature search and contributed to writing of the report. KW performed the statistical analysis. $S A$ and GR designed the figures. DCD, DF, WS, YC, ZNB, EL, and FL revised the manuscript for important intellectual content. All authors read and approved the final manuscript.

\section{Funding}

This work was supported by Grants G.0535.18N and 1524919N from the Research Fund (FWO) Flanders (Belgium), by a grant of the Baillet-Latour Fund, by a grant of the Belgian Foundation against Cancer (Stichting tegen Kanker) [2016-138 (FAF-C/2016/764)], by a grant of the Foundation Horlait-Dapsens, and by the Shanghai Rising-Star Program (18QA1401000). We also received support from the Belgian non-profit organisation Organisation for Medical Education \& Research vzw, from a Methusalem Fund from the University of Antwerp, from the Kaushik Bhansali Fund, and from Gilead Sciences and Janssen Pharmaceuticals. GR is supported by a Doctoral Grant Strategic Basic Research of the FWO (Grant 1S72821N), an Emmanuel van der Schueren fellowship from Kom op tegen Kanker (Stand up to Cancer, Belgium), and the public utility foundation MeToYou (Belgium). DCD is supported by a DOC-PRO PhD grant of the Special Research Fund (BOF) of the University of Antwerp and by Grant G053518N from the FWO. SA is a senior clinical investigator of the FWO and holder of the 2020 Gilead BeLux fellowship.

\section{Availability of data and materials}

All data generated or analyzed during this study are included in this published article [and its supplementary information files].

\section{Ethics approval and consent to participate}

Not applicable.

\section{Consent for publication}

Not applicable.

\section{Competing interests}

The authors declare that they have no competing interests.

\section{Author details}

${ }^{1}$ Laboratory of Experimental Hematology, Vaccine and Infectious Disease Institute, Faculty of Medicine and Health Sciences, University of Antwerp, Antwerp, Belgium. ${ }^{2}$ Division of Hematology and Center for Cell Therapy \& Regenerative Medicine, Antwerp University Hospital, Edegem, Belgium. ${ }^{3}$ Clinical Trial Center, Antwerp University Hospital, Edegem, Belgium. ${ }^{4}$ Biotherapy Research Center, Fudan University, Shanghai, China. ${ }^{5}$ Department of Digestive Diseases, Huashan Hospital of Fudan University, Shanghai, China.

Received: 11 October 2020 Accepted: 17 November 2020

Published online: 03 December 2020 


\section{References}

1. Bianchi G, Anderson KC. Understanding biology to tackle the disease: multiple myeloma from bench to bedside, and back. CA Cancer J Clin. 2014;64(6):422-44.

2. Rollig C, Knop S, Bornhauser M. Multiple myeloma. Lancet. 2015;385(9983):2197-208.

3. Dimopoulos MA, Terpos E. Multiple myeloma. Ann Oncol. 2010;21:vii143-50.

4. Turesson I, Bjorkholm M, Blimark CH, Kristinsson S, Velez R, Landgren O. Rapidly changing myeloma epidemiology in the general population: increased incidence, older patients, and longer survival. Eur J Haematol. 2018:101:237-44

5. Abramson HN. The multiple myeloma drug pipeline-2018: a review of small molecules and their therapeutic targets. Clin Lymphoma Myeloma Leuk. 2018;18(9):611-27.

6. Roex G, Feys T, Beguin Y, Kerre T, Poiré X, Lewalle $P$, et al. Chimeric antigen receptor-T-cell therapy for $\mathrm{B}$-cell hematological malignancies: an update of the pivotal clinical trial data. Pharmaceutics. 2020;12(2):194.

7. Wu C, Zhang L, Brockman QR, Zhan F, Chen L. Chimeric antigen receptor T cell therapies for multiple myeloma. J Hematol Oncol. 2019:12(1):120.

8. Cohen AD. CAR-T cells and other cellular therapies for multiple myeloma: 2018 update. Am Soc Clin Oncol Educ Book. 2018;38:e6-15.

9. Hartmann J, Schüßler-Lenz M, Bondanza A, Buchholz CJ. Clinical development of CART cells-challenges and opportunities in translating innovative treatment concepts. EMBO Mol Med. 2017:9(9):1183-97.

10. Danhof S, Hudecek M, Smith EL. CARs and other T cell therapies for MM: the clinical experience. Best Pract Res Clin Haematol. 2018:31(2):147-57.

11. Timmers M, Roex G, Wang Y, Campillo-Davo D, Van Tendeloo VFI, Chu Y, et al. Chimeric antigen receptor-modified T cell therapy in multiple myeIoma: beyond B cell maturation antigen. Front Immunol. 2019;10:1613.

12. Yu B, Jiang T, Liu D. BCMA-targeted immunotherapy for multiple myeloma. J Hematol Oncol. 2020;13(1):125.

13. Grupp SA, Kalos M, Barrett D, Aplenc R, Porter DL, Rheingold SR, et al. Chimeric antigen receptor-modified $T$ cells for acute lymphoid leukemia. N Engl J Med. 2013;368(16):1509-18.

14. Maude SL, Frey N, Shaw PA, Aplenc R, Barrett DM, Bunin NJ, et al. Chimeric antigen receptor $T$ cells for sustained remissions in leukemia. N Engl J Med. 2014:371(16):1507-17.

15. Wang Y, Luo F, Yang J, Zhao C, Chu Y. New chimeric antigen receptor design for solid tumors. Front Immunol. 2017;8:1934.

16. D'Agostino M, Raje N. Anti-BCMA CAR T-cell therapy in multiple myeloma: can we do better? Leukemia. 2020;34(1):21-34

17. Liu D, Zhao J. Cytokine release syndrome: grading, modeling, and new therapy. J Hematol Oncol. 2018;11(1):121

18. Gagelmann N, Ayuk F, Atanackovic D, Kröger N. B cell maturation antigenspecific chimeric antigen receptor $T$ cells for relapsed or refractory multiple myeloma: a meta-analysis. Eur J Haematol. 2020;104(4):318-27.

19. Garfall AL, Maus MV, Hwang WT, Lacey SF, Mahnke YD, Melenhorst $\mathrm{J}$, et al. Chimeric antigen receptor T cells against CD19 for multiple myeloma. N Engl J Med. 2015;373(11):1040-7.

20. Cohen AD, Garfall AL, Stadtmauer EA, Melenhorst JJ, Lacey SF, Lancaster $E$, et al. B cell maturation antigen-specific CART cells are clinically active in multiple myeloma. J Clin Invest. 2019:129(6):2210-21.

21. Cohen AD, Melenhorst JJ, Garfall AL, Lacey SF, Davis M, Vogl DT, et al. Predictors of $T$ cell expansion and clinical responses following B-cell maturation antigen-specific chimeric antigen receptor $T$ cell therapy (CART-BCMA) for relapsed/refractory multiple myeloma (MM). Blood. 2018:132:1974

22. Han L, Gao Q, Zhou K, Zhou J, Fang B, Zhang J, et al. The phase I clinical study of CART targeting BCMA with humanized alpaca-derived single-domain antibody as antigen recognition domain. J Clin Oncol. 2019;37:2535

23. Kumar S, Paiva B, Anderson KC, Durie B, Landgren O, Moreau P, et al. International myeloma working group consensus criteria for response and minimal residual disease assessment in multiple myeloma. Lancet Oncol. 2016:17(8):e328-46.

24. Raje N, Berdeja J, Lin Y, Siegel D, Jagannath S, Madduri D, et al. Anti-BCMA CAR T-cell therapy bb2121 in relapsed or refractory multiple myeloma. N Engl J Med. 2019;380(18):1726-37

25. Brudno JN, Maric I, Hartman SD, Rose JJ, Wang M, Lam N, et al. T cells genetically modified to express an anti-B-cell maturation antigen chimeric antigen receptor cause remissions of poor-prognosis relapsed multiple myeloma. J Clin Oncol. 2018;36(22):2267-80.

26. Mailankody S, Ghosh A, Staehr M, Purdon TJ, Roshal M, Halton E, et al. Clinical responses and pharmacokinetics of MCARH171, a humanderived bcma targeted CAR T cell therapy in relapsed/refractory multiple myeloma: final results of a phase I clinical trial. Blood. 2018;132:959.

27. Yan Z, Cao J, Cheng H, Qiao J, Zhang H, Wang Y, et al. A combination of humanised anti-CD19 and anti-BCMA CART cells in patients with relapsed or refractory multiple myeloma: a single-arm, phase 2 trial. Lancet Haematol. 2019;6(10):e521-9.

28. Raje N, Berdeja J, Lin Y, Munshi N, Siegel DSD, Liedtke M, et al. bb2121 anti-BCMA CAR T-cell therapy in patients with relapsed/refractory multiple myeloma: updated results from a multicenter phase I study. J Clin Oncol. 2018;36(15):8007.

29. Berdeja JG, Alsina M, Shah ND, Siegel DS, Jagannath S, Madduri D, et al. Updated results from an ongoing phase 1 clinical study of bb21217 antibcma CAR-T-cell therapy. Blood. 2019;134:927.

30. Xu J, Wang Q, Xu H, Gu C, Jiang L, Wang J, et al. Anti-BCMA CAR-T cells for treatment of plasma cell dyscrasia: case report on POEMS syndrome and multiple myeloma. J Hematol Oncol. 2018;11(1):128.

31. Li C, Wang Q, Zhu H, Mao X, Wang Y, Zhang Y, et al. T cells expressing anti $B$-cell maturation antigen chimeric antigen receptors for plasma cell malignancies. Blood. 2018;132:1013.

32. Ali SA, Shi V, Maric I, Wang M, Stroncek DF, Rose JJ, et al. T cells expressing an anti-B-cell maturation antigen chimeric antigen receptor cause remissions of multiple myeloma. Blood. 2016;128(13):1688-700.

33. Li C, Mei H, Hu Y, Guo T, Liu L, Jiang H, et al. A bispecific CAR-T cell therapy targeting bcma and CD38 for relapsed/refractory multiple myeloma: updated results from a phase 1 dose-climbing trial. Blood. 2019;134:930.

34. Jie J, Hao S, Jiang S, Li Z, Yang M, Zhang W, et al. Phase 1 trial of the safety and efficacy of fully human anti-bcma CART cells in relapsed/refractory multiple myeloma. Blood. 2019;134:4435.

35. Jiang $S$, Jin J, Hao S, Yang $M$, Chen $L$, Ruan $H$, et al. Low dose of human scFv-derived BCMA-targeted CAR-T cells achieved fast response and high complete remission in patients with relapsed/refractory multiple myeloma. Blood. 2018;132:960.

36. Mailankody S, Htut M, Lee KP, Bensinger W, Devries T, Piasecki J, et al. JCARH125, anti-BCMA CAR T-cell therapy for relapsed/refractory multiple myeloma: initial proof of concept results from a phase 1/2 multicenter study (EVOLVE). Blood. 2018;132:957.

37. Yao X, Zhu S, Huang J, Qu X, Zhu J, Wei Y, et al. Developing a novel anti-bcma CAR-T for relapsed or refractory multiple myeloma. Blood. 2019;134:50.

38. Li C, Wang J, Wang D, Hu G, Yang Y, Zhou X, et al. Efficacy and safety of fully human BCMA targeting CART cell therapy in relapsed/refractory multiple myeloma. Blood. 2019;134:929.

39. Garfall AL, Cohen AD, Lacey SF, Tian L, Hwang W-T, Vogl DT, et al. Combination anti-BCMA and anti-CD19 CAR T cells as consolidation of response to prior therapy in multiple myeloma. Blood. 2019;134:1863.

40. Green DJ, Pont M, Sather BD, Cowan AJ, Turtle CJ, Till BG, et al. Fully human BCMA targeted chimeric antigen receptor $T$ cells administered in a defined composition demonstrate potency at low doses in advanced stage high risk multiple myeloma. Blood. 2018:132:1011.

41. Cowan AJ, Pont M, Sather BD, Turtle CJ, Till BG, Nagengast AM, et al. Efficacy and safety of fully human BCMA CAR T cells in combination with a gamma secretase inhibitor to increase BCMA surface expression in patients with relapsed or refractory multiple myeloma. Blood. 2019:134:204

42. Yan L, Yan Z, Shang J, Shi X, Jin S, Kang L, et al. Sequential CD19- and BCMA-specific chimeric antigen receptor T cell treatment for RRMM: report from a single center study. Blood. 2019;134:578.

43. Shi X, Yan L, Shang J, Kang L, Jin S, Kang H, et al. Combined infusion of anti-CD19 and anti-BCMA CART cells after early or later transplantation in the front line was superior to salvage therapy for high risk MM. Blood. 2019;134:1949.

44. Mikkilineni L, Manasanch EE, Lam N, Vanasse D, Brudno JN, Maric I, et al. T cells expressing an anti-B-cell maturation antigen (BCMA) chimeric antigen receptor with a fully-human heavy-chain-only antigen recognition domain induce remissions in patients with relapsed multiple myeloma. Blood. 2019;134:3230 
45. Gregory T, Cohen AD, Costello CL, Ali SA, Berdeja JG, Ostertag EM, et al. Efficacy and safety of p-BCMA-101 CAR-T cells in patients with relapsed/ refractory ( $r / r)$ multiple myeloma (MM). Blood. 2018;132:1012.

46. Han L, Gao Q, Zhou K, Yin Q, Fang B, Zhou J, et al. Development and evaluation of CART targeting bema with humanized alpaca-derived singledomain antibody as antigen recognition domain. Blood. 2018;132:1976.

47. Xu J, Chen L-J, Yang S-S, Sun Y, Wu W, Liu Y-F, et al. Exploratory trial of a biepitopic CAR T-targeting B cell maturation antigen in relapsed/refractory multiple myeloma. Proc Natl Acad Sci U S A. 2019:116(19):9543-51.

48. Chen L, Xu J, Fu W Sr, Jin S, Yang S, Yan S, et al. Updated phase 1 results of a first-in-human open-label study of LCAR-B38M, a structurally differentiated chimeric antigen receptor T (CAR-T) cell therapy targeting B-cell maturation antigen (BCMA). Blood. 2019;134:1858.

49. Zhao W-H, Liu J, Wang B-Y, Chen Y-X, Cao X-M, Yang Y, et al. A phase 1, open-label study of LCAR-B38M, a chimeric antigen receptor T cell therapy directed against B cell maturation antigen, in patients with relapsed or refractory multiple myeloma. J Hematol Oncol. 2018a;11(1):141.

50. Wang B-Y, Zhao W-H, Liu J, Chen Y-X, Cao X-M, Yang Y, et al. Long-term follow-up of a phase 1, first-in-human open-label study of LCAR-B38M, a structurally differentiated chimeric antigen receptor $T$ (CAR-T) cell therapy targeting $\mathrm{B}$-cell maturation antigen (BCMA), in patients (pts) with relapsed/refractory multiple myeloma (RRMM). Blood. 2019;134:579.

51. Madduri D, Usmani SZ, Jagannath S, Singh I, Zudaire E, Yeh T-M, et al. Results from CARTITUDE-1: a phase 1b/2 study of JNJ-4528, a CAR-T cell therapy directed against B-cell maturation antigen (BCMA), in patients with relapsed and/or refractory multiple myeloma (R/R MM). Blood. 2019;134:577.

52. Hu Y, Yanlei Z, Wei G, Alex Hong C, Huang H. Potent anti-tumor activity of BCMA CAR-T therapy against heavily treated multiple myeloma and dynamics of immune cell subsets using single-cell mass cytometry. Blood. 2019:134:1859.

53. Fu W Sr, Du J, Jiang H, Cheng Z, Wei R, Yu K, et al. Efficacy and safety of CAR-T therapy with safety switch targeting bcma for patients with relapsed/refractory multiple myeloma in a phase 1 clinical study. Blood. 2019:134:3154.

54. Zhang H, Gao L, Liu L, Wang J, Wang S, Gao L, et al. A BCMA and CD19 bispecific CAR-T for relapsed and refractory multiple myeloma. Blood. 2019:134:3147.

55. Cho SF, Anderson KC, Tai YT. Targeting B cell maturation antigen (BCMA) in multiple myeloma: potential uses of BCMA-based immunotherapy. Front Immunol. 2018;9:1821.

56. Hermanson DL, Barnett BE, Rengarajan $S$, Codde $R$, Wang $X$, Tan $Y$, et al. A novel BCMA-specific, centyrin-based CAR-T product for the treatment of multiple myeloma. Blood. 2016;128(22):2127.

57. Zhao W-H, Liu J, Wang B-Y, Chen Y-X, Cao X-M, Yang Y, et al. Updated analysis of a phase 1, open-label study of LCAR-B38M, a chimeric antigen receptor $T$ cell therapy directed against B-cell maturation antigen, in patients with relapsed/refractory multiple myeloma. Blood. 2018b;132:955.

58. Shah N, Chari A, Scott E, Mezzi K, Usmani SZ. B-cell maturation antigen (BCMA) in multiple myeloma: rationale for targeting and current therapeutic approaches. Leukemia. 2020;34(4):985-1005.
59. Munshi NC, Anderson JLD, Shah N, Jagannath S, Berdeja JG, Lonial S, et al. Idecabtagene vicleucel (ide-cel; bb2121), a BCMA-targeted CAR T-cell therapy, in patients with relapsed and refractory multiple myeloma (RRMM): initial KarMMa results. J Clin Oncol. 2020;38:8503.

60. Gust J, Hay KA, Hanafi L-A, Li D, Myerson D, Gonzalez-Cuyar LF, et al. Endothelial activation and blood-brain barrier disruption in neurotoxicity after adoptive immunotherapy with CD19 CAR-T cells. Cancer Discov. 2017;7(12):1404-19.

61. Santomasso BD, Park JH, Salloum D, Riviere I, Flynn J, Mead E, et al. Clinical and biological correlates of neurotoxicity associated with CAR T-cell therapy in patients with B-cell acute lymphoblastic leukemia. Cancer Discov. 2018;8(8):958-71.

62. Fraietta JA, Lacey SF, Orlando EJ, Pruteanu-Malinici I, Gohil M, Lundh S, et al. Determinants of response and resistance to CD19 chimeric antigen receptor (CAR) T cell therapy of chronic lymphocytic leukemia. Nat Med. 2018;24(5):563-71.

63. Dancy E, Garfall AL, Cohen AD, Fraietta JA, Davis M, Levine BL, et al. Clinical predictors of T cell fitness for CART cell manufacturing and efficacy in multiple myeloma. Blood. 2018;132:1886.

64. Strohl WR, Naso M. Bispecific T-cell redirection versus chimeric antigen receptor (CAR)-T cells as approaches to kill cancer cells. Antibodies. 2019;8(3):41.

65. Lam N, Trinklein ND, Buelow B, Patterson GH, Ojha N, Kochenderfer JN. Anti-BCMA chimeric antigen receptors with fully human heavy-chainonly antigen recognition domains. Nat Commun. 2020;11(1):283.

66. Ying Z, He T, Wang X, Zheng W, Lin N, Tu M, et al. Parallel comparison of 4-1BB or CD28 co-stimulated CD19-targeted CAR-T cells for B cell nonHodgkin's lymphoma. Mol Ther Oncolytics. 2019;15:60-8.

67. Jagannath S, Lin Y, Goldschmidt H, Reece DE, Nooka AK, Otero PR, et al. KarMMa-RW: a study of real-world treatment patterns in heavily pretreated patients with relapsed and refractory multiple myeloma (RRMM) and comparison of outcomes to KarMMa. J Clin Oncol. 2020;38:8525.

68. Shah N, Alsina M, Siegel DS, Jagannath S, Madduri D, Kaufman JL, et al. Initial results from a phase 1 clinical study of bb21217, a next-generation anti bcma CART therapy. Blood. 2018;132:488.

69. Hamieh M, Dobrin A, Cabriolu A, van der Stegen SJC, Giavridis T, MansillaSoto J, et al. CART cell trogocytosis and cooperative killing regulate tumour antigen escape. Nature. 2019;568(7750):112-6.

70. Berahovich R, Liu X, Zhou H, Tsadik E, Xu S, Golubovskaya V, et al. Hypoxia selectively impairs CAR-T cells in vitro. Cancers. 2019;11(5):602.

71. Bernabei L, Garfall AL, Melenhorst JJ, Lacey SF, Stadtmauer EA, VogI DT, et al. PD-1 inhibitor combinations as salvage therapy for relapsed/refractory multiple myeloma (MM) patients progressing after BCMA-directed CAR-T Cells. Blood. 2018;132:1973.

\section{Publisher's Note}

Springer Nature remains neutral with regard to jurisdictional claims in published maps and institutional affiliations.

Ready to submit your research? Choose BMC and benefit from

- fast, convenient online submission

- thorough peer review by experienced researchers in your field

- rapid publication on acceptance

- support for research data, including large and complex data types

- gold Open Access which fosters wider collaboration and increased citations

- maximum visibility for your research: over $100 \mathrm{M}$ website views per year

At BMC, research is always in progress.

Learn more biomedcentral.com/submissions 